\title{
Multipacket Reception in Wireless Local Area Networks ${ }^{*}$
}

\author{
Peng Xuan Zheng, Ying Jun (Angela) Zhang and Soung Chang Liew \\ Department of Information Engineering \\ The Chinese University of Hong Kong \\ Shatin, New Territories, Hong Kong \\ \{pxzheng4, yjzhang, soung\}@ie.cuhk.edu.hk
}

\begin{abstract}
The conventional MAC (Medium Access Control) protocols assume that only one packet can be received at a given time. However, with the advent of sophisticated signal processing and antenna array techniques, it is possible to achieve multipacket reception (MPR) in the physical layer (PHY). In this paper, we propose a PHY methodology and the corresponding MAC protocol for MPR in wireless local area networks (WLANs). The proposed MAC protocol closely follows the 802.11 DCF (Distributed Coordination Function) scheme and enables MPR in a distributed manner. For the proposed MPR system, a closed-form expression of the average throughput is derived. Based on the expression, an optimal transmission probability that maximizes the throughput can be attained. In addition, two enhancement schemes are presented to further improve the performance of the MPR protocol. Numerical results show that the proposed MPR system can considerably increase the spectrum efficiency compared to the WLANs with conventional collision models.
\end{abstract}

Index Terms - WLAN, 802.11, MPR, cross-layer design.

\section{INTRODUCTION}

Wireless local area networks (WLANs) have received much attention these years from both the academic and industry fields. Thanks to its simplicity and cost-efficiency, IEEE 802.11-based WLAN shows unique advantages in providing high-speed and low cost wireless services in hot spots and indoor environments [1]. As the need for broadband multimedia communications grows explosively, it is increasingly important for 802.11 to support higher spectrum efficiency and network capacity. Besides achieving a high data rate at the physical layer (PHY), it is essential for the MAC (Medium Access Control) protocols to fully exploit the benefits provided by advanced PHY techniques so that an overall high MAC throughput is achieved [2].

In conventional 802.11 WLAN, packets transmitted at the same time are assumed to be destroyed due to collision. Thanks to the advanced signal processing techniques developed recently, the single packet reception constraint in the PHY layer can be relaxed. In the infrastructure mode, for example, packets transmitted at the same time can be jointly decoded at the AP by MUD (Multiuser Detection) techniques when the AP is equipped with multiple antennas. By exploiting the MPR (Multipacket Reception) capability at the PHY layer, packet transmissions are less restrained than the

\footnotetext{
* This work was sponsored by the Areas of Excellence scheme established under the University Grant Committee of the Hong Kong Special Administrative Region, China (Project Number AoE/E-01/99).
}

conventional medium access protocols. Consequently, an increase in the system throughput is expected.

The concept of MPR was first put forward by S. Ghez et al [3]. However, research on MPR is still very limited. This is partly due to the need for cross-layer design, which requires signal processing at the PHY layer as well as protocol design at the MAC layer. Most of the existing MPR MAC protocols $[4,5]$ assume the existence of a central coordinator that schedules transmissions. Hence, these protocols do not apply to the 802.11-based WLANs due to the absence of a central controller in the most commonly deployed DCF (Distributed Coordination Function) mode.

In this paper, we propose a novel MAC protocol as well as a PHY methodology to implement MPR in 802.11. The proposed protocol closely follows the 802.11 DCF scheme, with moderate modifications on the current MAC and PHY designs. To the best of our knowledge, our paper is the first to attempt MPR in a distributed manner.

We present a theoretical analysis framework for the MPR MAC protocol. A closed-form throughput expression is derived for the proposed MPR system. Based on the expression, we then calculate the optimal transmission probability that maximizes the throughput. In addition, two enhancement approaches that further improve the performance of the proposed MPR system are introduced. Our results show that the proposed system considerably improves the throughput and spectrum efficiency compared to the WLANs with conventional collision models.

The rest of this paper is organized as follows. Section II discusses the methodology in the physical layer for MPR support. Section III proposes the novel MAC protocol for MPR. Section IV derives the closed-form throughput expression for MPR and shows the results of the numerical analysis. Section V briefly introduces two other methods as enhancements to further improve the performance of the proposed MAC protocol for MPR. Finally, Section VI concludes the paper.

\section{Physical Layer Methodology}

In the rest of the paper, we restrict our considerations to the uplink (i.e., from client stations to AP) of an isolated BSS (Basic Service Set) for simplicity. Due to the space limitation, the downlink counterpart, which involves MPT (Multipacket Transmission), will be discussed in our future works. By "isolated", we mean the concerned BSS is sufficiently far away from other BSSs such that there is no overlap or interference between them. The system configuration is 
depicted in Fig. 1. The AP is mounted with $M$ antennas, while each client station has one antenna only.

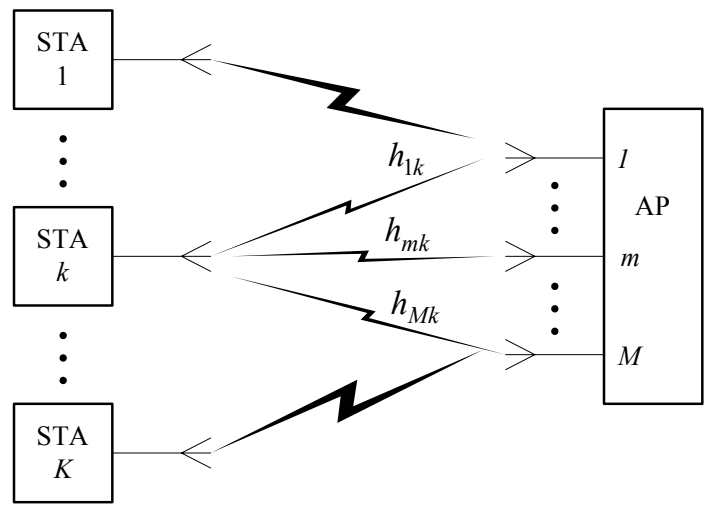

Fig. 1 System model used for MPR.

In this section, we propose a mechanism to implement MPR in 802.11. The basic idea is as follows. Client stations transmit RTS packets according to the 802.11 protocol. Note that it is possible for more than one RTS packets to be transmitted at the same time. Since the AP has no priori knowledge of who the senders are as well as the CSI (Channel State Information) on the corresponding links, MUD techniques such as ZF (Zero Forcing) and MMSE (Minimum Mean Squared Error) cannot be directly applied. Fortunately, the RTS packets are typically transmitted at a lower data rate than the data packets in 802.11. This setting is particularly suitable for blind detection algorithms, such as CM (Constant Modulus) or FA (Finite Alphabet), to separate the multiple RTS packets with reasonable computational complexity [6, 7].

In our proposed system, a FA-based blind detection scheme is applied to decode the RTS packets that are simultaneously transmitted from $K$ stations for $K \leq M$. If $K>M$, the sources are no longer identifiable and collisions are incurred. Upon successfully decoding the RTS packets, the AP can then identify the senders of the packets. Training sequences, which are to be transmitted in the preamble of the data packets, are then allocated to these users to facilitate channel estimation during the data transmission phase. Since the $K$ stations will transmit their data packets at the same time, their training sequences should be mutually orthogonal. In our system, at most $M$ simultaneous transmissions are allowed, since there are $M$ antennas at the AP. Therefore, a total of $M$ orthogonal sequences are required to be predefined and known to all stations in the BSS. The sequence allocation decision is sent to the users via the CTS packet.

During the data transmission phase, CSI is estimated from the orthogonal training sequences that are transmitted in the preamble of the data packets. With the estimated CSI, various MUD techniques can be applied to separate the multiple data packets at the AP. Thanks to the coherent detection, data packets can be transmitted at a much higher rate than the RTS packets without involving excessive computational complexity.

The details of the PHY realization of MPR are presented in the following subsections.

\section{A. Blind RTS Separation}

Assume that the delay spread is smaller than the symbol duration, and hence the effect of the channel is approximated by a complex amplitude scaling. Let $h_{m, k}$ denote the channel coefficient from user $k$ to the $m^{\text {th }}$ receive antenna and $x_{k}(n)$ denote the symbol transmitted by user $k$ over symbol duration $n$. The received signals can then be written as

$$
\mathbf{y}(n)=\left[y_{1}(n), y_{2}(n), \cdots, y_{M}(n)\right]^{T}=\mathbf{H x}(n)+\mathbf{w}(n)
$$

where

$$
\begin{gathered}
\mathbf{H}=\left[\begin{array}{cccc}
h_{1,1} & h_{1,2} & \cdots & h_{1, K} \\
h_{2,1} & h_{2,2} & \cdots & h_{2, K} \\
\vdots & \vdots & \ddots & \vdots \\
h_{M, 1} & h_{M, 2} & \cdots & h_{M, K}
\end{array}\right], \\
\mathbf{x}(n)=\left[x_{1}(n), x_{2}(n), \cdots, x_{K}(n)\right]^{T},
\end{gathered}
$$

and $\mathbf{w}(n)$ is the additive white noise received in the $n$th symbol duration. In an indoor environment, large angular spread is typically observed at the AP. Therefore, the entries in the channel matrix $\mathbf{H}$ are modelled as i.i.d. complex Gaussian random variables.

Assuming that the channel is constant over an RTS packet, which is composed of $N$ symbol periods, we obtain the following block formulation of the data

$$
\mathbf{Y}=\mathbf{H X}-\mathbf{W}
$$

where $\mathbf{Y}=[\mathbf{y}(1), \mathbf{y}(2), \cdots \mathbf{y}(N)], \mathbf{X}=[\mathbf{x}(1), \mathbf{x}(2), \cdots \mathbf{x}(N)]$, and $\mathbf{W}=[\mathbf{w}(1), \mathbf{w}(2), \cdots \mathbf{w}(N)]$. The problem to be addressed here is the estimation of the number of sources $K$, the channel matrix $\mathbf{H}$, and the symbol matrix $\mathbf{X}$, given the array output $\mathbf{Y}$.

\section{1) Estimation of the number of sources $K$}

To begin with, we ignore the white noise for the moment and have $\mathbf{Y}=\mathbf{H X}$. The rank of $\mathbf{H}$ is equal to $K$ if $K \leq M$. Likewise, $\mathbf{X}$ is full-row-rank when $N$ is much larger than $K$. Consequently, we have

$$
\operatorname{rank}(\mathbf{Y})=K
$$

Let

$$
\mathbf{Y}=\mathbf{U S V}^{H},
$$

be the singular value decomposition of $\mathbf{Y}$, where $\mathbf{U}$ and $\mathbf{V}$ are matrices consisting of left and right singular vectors respectively, $\mathbf{S}$ is a diagonal matrix with the diagonal elements being the singular values, and $(\cdot)^{H}$ denotes the complex conjugate transpose of the matrix. Then, $K$ is equal to the number of nonzero entries in the diagonal of $\mathbf{S}$. With white noise added to the data, $K$ can be estimated from the number of singular values of $\mathbf{Y}$ (i.e., diagonal entries of $\mathbf{S}$ ) that are significantly larger than zero.

\section{2) Estimation of $\boldsymbol{X}$ and $\boldsymbol{H}$}

The maximum-likelihood estimator yields the following separable least-squares minimization problem [7]

$$
\min _{\mathbf{H}, \mathbf{X} \in \Omega}\|\mathbf{Y}-\mathbf{H X}\|_{F}^{2}
$$

where $\Omega$ is the finite alphabet to which the elements of $\mathbf{X}$ belong, and \|\|$_{F}^{2}$ is the Frobenius norm. The minimization of 
(7) can be carried out in two steps. First, we minimize (7) with respect to $\mathbf{H}$ and obtain

$$
\hat{\mathbf{H}}=\mathbf{Y} \mathbf{X}^{+}=\mathbf{Y} \mathbf{X}^{H}\left(\mathbf{X X}^{H}\right)^{-1},
$$

where $(.)^{+}$is the pseudo-inverse of a matrix. Substituting $\hat{\mathbf{H}}$ back into (7), we obtain a new criterion, which is a function of X only:

$$
\min _{\mathbf{X} \in \Omega}\left\|\mathbf{Y} \mathbf{P}_{\mathbf{X}^{H}}^{\perp}\right\|_{F}^{2},
$$

where $\mathbf{P}_{\mathbf{X}^{H}}^{\perp}=\mathbf{I}-\mathbf{X}^{H}\left(\mathbf{X X}^{H}\right)^{-1} \mathbf{X}$, and $\mathbf{I}$ is the identity matrix. The global minimum of (9) can be obtained by enumerating over all possible choices of $\mathbf{X}$. This search has an exponential complexity $L^{N K}$, where $L$ is the cardinality of $\Omega$ (i.e., constellation size). Reduced-complexity iterative algorithms such as ILSP and ILSE were introduced in [8]. The idea is to visit the received data iteratively until the best fit with the channel and signal model is obtained. Due to the space limitation, the details of ILSP and ILSE are not covered in this paper. Interested readers are referred to [8] and the references therein.

\section{B. Data Packet Detection}

After successfully decoding the RTS packets at the AP, the orthogonal training sequences are allocated to the requesting stations through the CTS packet. Given the orthogonal training sequences, the CSI in the data transmission phase can be estimated more accurately. We omit the index $n$ in this subsection, since the following processing is on a per-symbol basis. At a given symbol period, the received vector is

$$
\mathbf{y}=\sum_{k=1}^{K} \mathbf{h}_{k} x_{k}+\mathbf{w}=\mathbf{H x}+\mathbf{w},
$$

where $\mathbf{h}_{k}=\left[h_{1, k}, h_{2, k}, \cdots, h_{M, k}\right]^{T}$. To separate the signals from multiple users, various MUD techniques have been proposed in the literature. For example, the ZF (Zero Forcing) receiver is one of the most popular linear detectors. It multiplies the received vector by a decorrelation matrix $\mathbf{H}^{+}$, and the decision statistics become

$$
\mathbf{r}^{Z F}=\mathbf{H}^{+} \mathbf{y}=\mathbf{x}+\mathbf{H}^{+} \mathbf{w} .
$$

In contrast, the MMSE (Minimum Mean Square Error) receiver takes into account both the co-channel interference and the noise term. Such a receiver is the optimal linear detector in the sense of maximizing the SINR (Signal to Interference and Noise Ratio). The decision statistics are given by

$$
\mathbf{r}^{M M S E}=\left(\mathbf{H} \mathbf{H}^{H}+\sigma^{2} \mathbf{I}\right)^{-1} \mathbf{H}^{H} \mathbf{y} .
$$

Given the decision statistics, an estimate of $x_{k}$ can be obtained by feeding the $k^{\text {th }}$ element of $\mathbf{r}^{Z F}$ or $\mathbf{r}^{M M S E}$ into a quantizer.

\section{MAC PROTOCOL FOR MPR}

The proposed protocol follows the 802.11 DCF RTS/CTS (Request To Send/Clear To Send) access mechanism closely, with extension to support MPR. We describe the proposed protocol in this section. For simplicity, we use the same assumptions as in Section II (i.e., a single isolated BSS with an AP and $n$ associated client stations). We assume that the AP is the only station in the BSS with the capability to receive up to $M(M \geq 1)$ packets simultaneously.

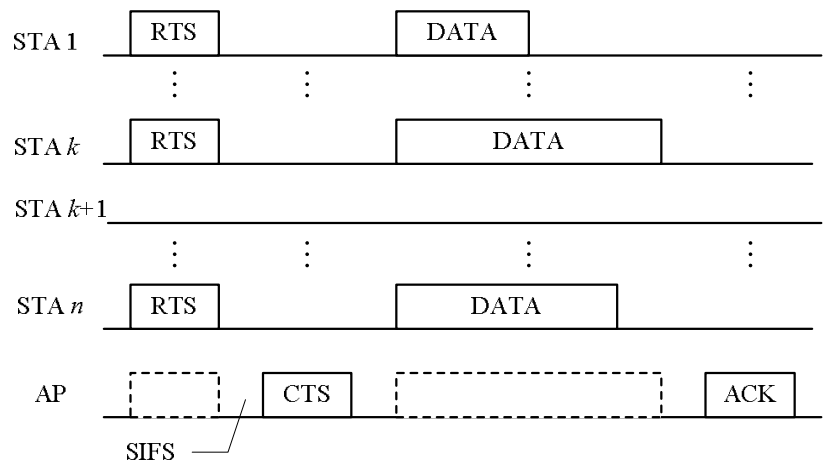

Fig. 2 Time line example for the MPR MAC.

Figure 2 illustrates the protocol operation. A STA (station) with a packet to transmit first sends an RTS frame to the AP. In our MPR MAC model, when multiple STAs transmit RTS frames at the same time, the AP can successfully detect all the RTS frames if and only if the number of RTSs is no more than $M$. When the number of transmitting STAs exceeds $M$, collisions occur and the AP cannot decode any of the RTSs .The STAs will retransmit their RTS frames after a backoff time period as the original 802.11 protocol.

When the AP detects the RTSs successfully, it responds, after a SIFS period, with a CTS frame that grants transmission permissions to all the requesting STAs. Then the transmitting STAs will start transmitting DATA frames after a SIFS, and the AP will acknowledge the reception of the DATA frames by an ACK frame.

Figure 3 shows the formats of the control frames for the proposed MPR MAC. The formats of the RTS and Data frames are the same as those defined in 802.11, while the CTS and ACK frames have been modified to accommodate multiple transmitting STAs for MPR. The fields from RA (Receiver Address) 1 to RA $M$ of the CTS frame are copied from the TA (Transmitter Address) field of the preceding RTS frames. If $K(K<M)$ STAs send RTS frames simultaneously requesting transmissions to the AP, then the fields from RA 1 to RA $K$ are active and contain the proper address information, while the remaining RA $K+1$ to RA $M$ fields are inactive and stuffed with some null values.

The orthogonal training sequence allocation decision can be inferred implicitly from the RA 1 and RA $M$ fields. For example, the transmitting STA of address RA $k$ can be assigned the predefined orthogonal sequence $k$. So the sequence allocation information can be conveyed from the order of the $M$ addresses.

The format of the ACK frame is similar to that of the CTS frame, and the RA fields contain the addresses of the STAs to be acknowledged.

The virtual carrier sensing mechanism of 802.11 is still largely applicable to MPR MAC. As in 802.11, STAs shall update their NAV according to the information received in the Duration/ID field of a valid frame, but only when the new 
NAV value is greater than the current NAV value and only when the frame is not addressed to the receiving STA. A major change is for the AP to set the Duration field of CTS to the longest durations of all the STAs granted permission to transmit. This ensures other STAs in the same BSS can update their NAV correctly.

\begin{tabular}{|c|c|c|c|c|c|c|}
\hline Octets: 2 & 2 & 6 & 6 & \multicolumn{2}{|c|}{4} & \\
\hline $\begin{array}{l}\text { Frame } \\
\text { Control }\end{array}$ & Duration & RA & TA & \multicolumn{2}{|c|}{ FCS } & \\
\hline \multicolumn{7}{|c|}{ MAC Header } \\
\hline Octets: 2 & 2 & 6 & 6 & $\ldots$ & 6 & 4 \\
\hline $\begin{array}{l}\text { Frame } \\
\text { Control }\end{array}$ & Duration & RA 1 & RA 2 & $\ldots$ & $\mathrm{RA} M$ & FCS \\
\hline \multicolumn{7}{|c|}{$\begin{array}{l}\text { MAC Header } \\
\text { (b) CTS frame }\end{array}$} \\
\hline Octets: 2 & 2 & 6 & 6 & $\ldots$ & 6 & 4 \\
\hline $\begin{array}{l}\text { Frame } \\
\text { Control }\end{array}$ & Duration & RA 1 & RA 2 & $\ldots$ & $\mathrm{RA} M$ & FCS \\
\hline
\end{tabular}

Fig. 3 Formats of control frames for the MPR MAC.

\section{PERformance ANAlysis}

In the following analysis, we assume the number of contending STAs is fixed and that all STAs operate in saturated conditions (i.e., they always have packets available for transmission). We also assume that all STAs can hear each other (i.e., there is no hidden terminals) and the channel is perfect in that there is no packet loss due to fading.

\section{A. Throughput Derivation}

We define the term slot time as the time interval between two consecutive backoff time counter decrements [9]. The slot time we define here is not necessarily the constant slot time size $\sigma$. In $802.11 \mathrm{DCF}$, the backoff counter will be frozen when the channel is sensed busy. We define our slot time to include the frozen time and it is variable.

Suppose there are $n$ STAs contending for channel access, and each transmits with probability $\tau$ in a given slot time. Let $P_{t r}$ be the probability that there is at least one transmission in the slot time. Then

$$
P_{t r}=1-(1-\tau)^{n} \text {. }
$$

The conditional probability $P_{s 1}$ that a single-packet transmission is successful is given by the probability that only one STA transmits, conditioning on the fact that there is at least one transmission,

$$
P_{s 1}=n \tau(1-\tau)^{n-1} / P_{t r} .
$$

Generally, let $P_{s k}$ be the probability that a $k$-packet simultaneous transmission is successful. It is given by the probability that $k$ STAs transmit, conditioning on the fact that there is at least one transmission,

$$
P_{s k}=\left(\begin{array}{l}
n \\
k
\end{array}\right) \tau^{k}(1-\tau)^{n-k} / P_{t r} .
$$

We define the throughput $S$ to be the ratio of payload information bits being transmitted and the total amount of time spent to successfully transmit the payload. Therefore,

$$
\begin{aligned}
S & =\frac{E[\text { payload information bits transmitted in a slot time }]}{E[\text { length of a slot time }]} \\
& =\frac{\sum_{k=1}^{M} k P_{s k} P_{t r} E[L]}{\left(1-P_{t r}\right) \sigma+\sum_{k=1}^{M} P_{s k} P_{t r} T_{s k}+P_{t r}\left(1-P_{s}\right) T_{c}}
\end{aligned}
$$

where $E[L]$ is the average payload length in bits, $T_{s k}$ is the average slot time spent when there are successful $k$-packet transmissions, $T_{c}$ is the average slot time when there are collisions, and $P_{s}=\sum_{k=1}^{M} P_{s k}$ is the conditional probability of successful transmissions in a busy time slot. The general throughput expression (16) derived for MPR also incorporates the 802.11 case. In particular, when $M=1$, the MPR throughput reduces to the 802.11 throughput.

In our protocol, the RTS/CTS access scheme is employed. Therefore

$$
\left\{\begin{aligned}
T_{s k}= & R T S+S I F S+\delta+C T S+S I F S+\delta+H \\
& +E\left[L_{k}^{*}\right] / R+S I F S+\delta+A C K+D I F S+\delta, \\
T_{c}= & R T S+D I F S+\delta
\end{aligned}\right.
$$

where $\delta$ is the propagation delay, $H=P H Y_{h d r}+M A C_{h d r}$ is the total overhead time to transmit the packet headers, $E\left[L_{k}^{*}\right]$ is the average length (in bits) of the longest payload involved in a $k$-packet simultaneous transmission, and $R$ is the data rate for payload transmission. In the following numerical investigations, we assume all packets have the same fixed length, i.e., the average length $E\left[L_{k}^{*}\right]=E[L]=L(1 \leq k \leq M)$ and $T_{s k}=T_{s}$, where $L$ and $T_{s}$ are constants.

It is clear from (16) that $S$ is a function of $\tau$. Therefore, the optimal value of $\tau$, which maximizes $S$, can be obtained by solving the equation

$$
d S / d \tau=0
$$

From the Markov model established in [9], the following two relations can be found:

$$
\tau=\frac{2(1-2 p)}{(1-2 p)(W+1)+p W\left(1-(2 p)^{m}\right)},
$$

and

$$
p=1-\sum_{k=0}^{M-1}\left(\begin{array}{l}
n-1 \\
k
\end{array}\right) \tau^{k}(1-\tau)^{n-1-k} .
$$

In the above, $p$ is the conditional collision probability, $W$ is the minimum contention window size, and $m$ is the maximum backoff stage. A close observation of (19) and (20) indicates that $\tau$ can be expressed as a function of $W$. In fact, as a special case, when $m=0$, i.e., no exponential backoff, a closed-form relationship between $\tau$ and $W$ is given by 


$$
\tau=\frac{2}{W-1} .
$$

Consequently, the transmission probability $\tau$ can be adjusted to the optimal value by changing the contention window size $W$. By doing so, an overall maximum throughput is achieved.

\section{B. Numerical Analysis}

In this section, the performance of the proposed MPR system is illustrated. Figure 4 depicts the throughput as a function of the transmission probability $\tau$ for both $802.11 \mathrm{~g}$ and MPR. The system parameters and other parameters we used are as listed in Table I. Assume that all the packets have constant payload length. In the MPR case, we assume $M=2$, i.e., the maximum number of packets the AP can receive simultaneously is equal to two, and the control frames (i.e., RTS, CTS and ACK) are as defined in our MPR protocol.

From Fig. 4, we can see a great increase (about $45 \%$ gain when $n=10$ ) in the maximum throughput of our protocol with MPR compared to the 802.11g. As shown in the above analysis, it is always possible to reach the maximum throughput by carefully adjusting the contention window size $W$.

Figure 5 shows the maximum throughput of MPR as $M$ varies. Here, we assume the number of stations $n=50$, and other parameters as listed in Table I. From Fig. 5, we conclude that the maximum throughput increases roughly linearly with $M$, and is not bounded by the physical layer data rates. In other words, as far as only the MAC layer is concerned (without considering other physical restrictions), maximum throughput is infinitely scalable with $M$. This feature of MPR is extremely attractive, because it breaks the conventional assumption that the MAC layer throughput can not exceed the physical layer data rates. Consequently, MPR is a promising technique for future wireless LANs to achieve a high MAC throughput, even without an underlying high data rate $\mathrm{PHY}$.

TABLE I

SYSTEM PARAMETERS AND ADDITIONAL PARAMETERS USED IN NUMERICAL

\begin{tabular}{|c|c|c|}
\hline \multicolumn{1}{|c|}{ ANALYSIS } & $802.11 \mathrm{~g}$ & MPR $(\mathrm{M}=2)$ \\
\hline Packet payload & $8184 \mathrm{bits}$ & $8184 \mathrm{bits}$ \\
\hline MAC header & $272 \mathrm{bits}$ & $272 \mathrm{bits}$ \\
\hline PHY overhead & $26 \mu \mathrm{s}$ & $26 \mu \mathrm{s}$ \\
\hline ACK & 112 bits + PHY & 160 bits + PHY \\
\hline RTS & 160 bits + PHY & 160 bits + PHY \\
\hline CTS & 112 bits + PHY & 160 bits + PHY \\
\hline Basic rate & $6 \mathrm{Mbps}$ & $6 \mathrm{Mbps}$ \\
\hline Data rate & $54 \mathrm{Mbps}$ & $54 \mathrm{Mbps}$ \\
\hline Slot time $c$ & $9 \mu \mathrm{s}$ & $9 \mu \mathrm{s}$ \\
\hline SIFS & $10 \mu \mathrm{s}$ & $10 \mu \mathrm{s}$ \\
\hline DIFS & $28 \mu \mathrm{s}$ & $28 \mu \mathrm{s}$ \\
\hline Propagation delay $\delta$ & $1 \mu \mathrm{s}$ & $1 \mu \mathrm{s}$ \\
\hline
\end{tabular}

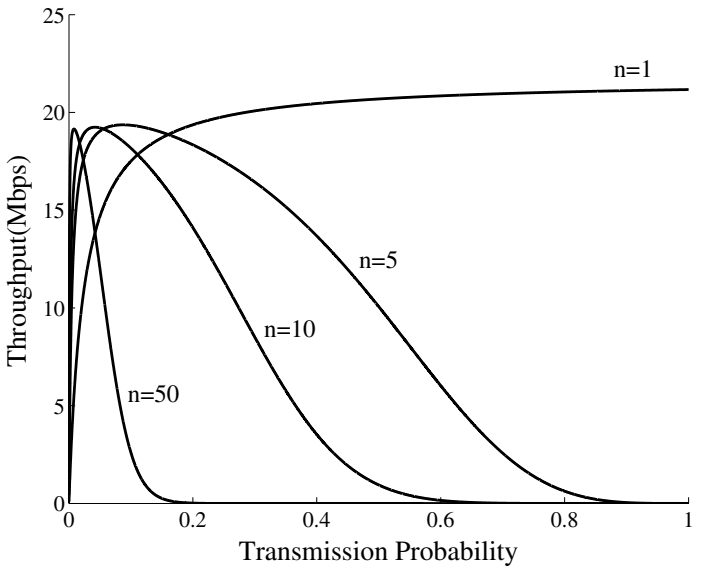

(a) $802.11 \mathrm{~g}$

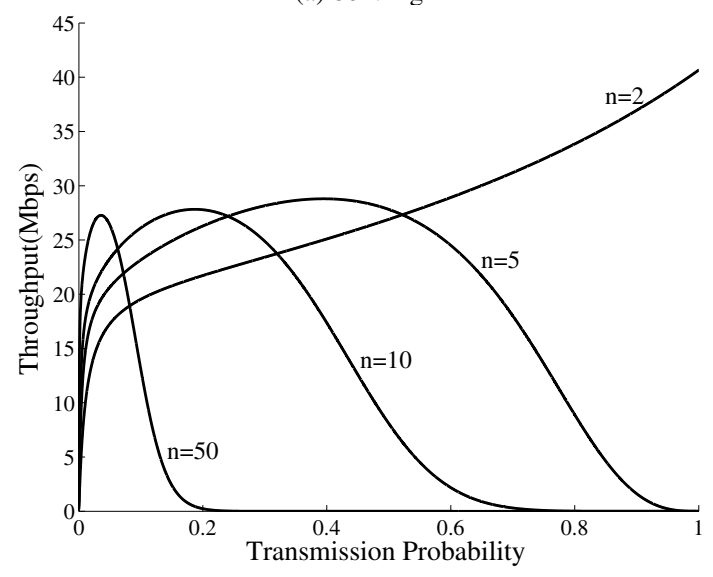

(b) MPR

Fig. 4 Throughput versus the transmission probability $\tau$ for $802.11 \mathrm{~g}$ and

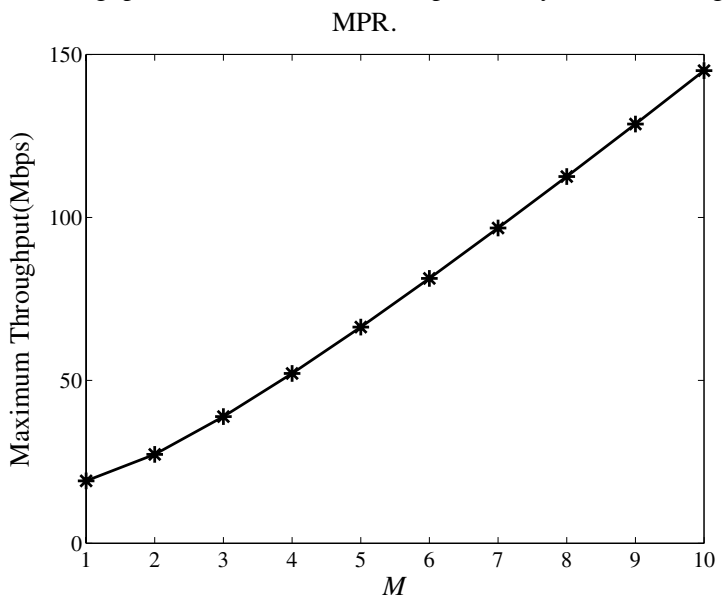

Fig. 5 Maximum throughput of MPR as a function of $M$

\section{ENHANCEMENTS OF THE MPR MAC}

Compared with the legacy 802.11 , the huge increase in maximum throughput by MPR is rather promising. This is achieved by increasing the number of antennas at the AP with ordinary STA having just one antenna, and a moderate modification in the MAC. However, there is still room for further improvement. Two possible MPR enhancement protocols are discussed in this section.

\section{A. Two-Round RTS Contention (TRRC)}


Theoretically an AP with $M$ antennas is capable of receiving up to $M$ packets at the same time. However, with the same backoff scheme as used in 802.11, it cannot be expected to always receive $M$ packets simultaneously. This is part of the reason why the maximum throughput with MPR is not $M$ times that of 802.11. This observation implies that precious resources are wasted during time slots with less than $M$-packet receptions.

In TRRC (Fig. 6), the STAs ready to transmit RTS will transmit with probability $p$ in the first contention round, and the remainders transmit in the second round. With reference to the analysis in Section IV, instead of transmitting with probability $\tau$ in a given slot, a STA in TRRC transmits with probability $p \tau$ in the first contention round. Therefore, on average, $n p \tau$ STAs transmit in the first round, and the remaining $n(1-p) \tau$ STAs transmit in the second round.

With TRRC, the chances that more STAs win the contention increase, and hence a higher throughput is expected. For instance, with $M=2, n=50, p=0.5$ and other parameters found in Table I, the maximum throughput of TRRC outperforms that of the original MPR MAC by $19 \%$.

\section{B. Adaptive Transmission Rate Control (ATRC)}

Thanks to the receive diversity at the AP, when the number of transmitting STAs $K<M$, we can increase the transmission rate per user. The basic idea of this approach is to control the transmission rate of the data frames adaptively, according to the number of simultaneous transmitters. When $K<M$, we make use of the receive diversity to transmit the data frames at higher rates.

In this case, we have

$$
\begin{aligned}
T_{s k}= & R T S+S I F S+\delta+C T S+S I F S+\delta+H+ \\
& E\left[L_{k}^{*}\right] / R_{k}+S I F S+\delta+A C K+D I F S+\delta
\end{aligned} .
$$

where $R_{k}$ is the data rate of a $k$-packet simultaneous transmission. Therefore, in this ATRC scheme, the value of $T_{s k}$ depends explicitly on the data rate adopted in a $k$-packet transmission.

For example, when $M=2, n=50, R_{1}=1.25 \mathrm{Mbps}$ and $R_{2}=1 \mathrm{Mbps}$, there is about $7 \%$ increase in the maximum throughput compared with the original MPR protocol

\begin{tabular}{|c|c|c|c|c|}
\hline \multirow[t]{2}{*}{ STA 1} & RTS & & & DATA \\
\hline & $\vdots$ & $\vdots$ & $\vdots$ & $\vdots$ \\
\hline STA $k$ & & RTS & & DATA \\
\hline
\end{tabular}
proposed in Section III.

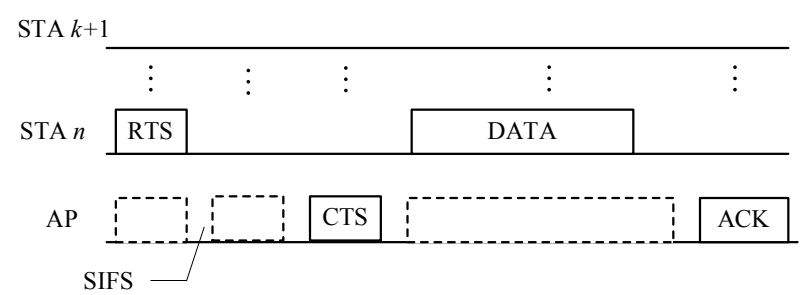

Fig. 6 Time line example for the TRRC scheme.

\section{CONCLUSION}

The conventional distributed multiple-access protocols assume that collisions happen when more than one packet is transmitted. The concept of MPR, however, gives rise to the possibility of multiple-packet reception without collisions.

This paper has proposed and investigated the PHY implementation and MAC protocols of an 802.11-like wireless network that incorporates MPR. In addition, analysis has been provided to evaluate the system performance. Specifically, a general throughput expression has been derived for system in which the access point uses $M>1$ antennas and the client stations use just one antenna. This baseline system allows up to $M$ packets to be received by the AP without collisions.

Our results show that about $45 \%$ increase (when $n=10$ ) in maximum throughput is possible when there are two antennas at the AP, assuming similar system parameters as in $802.11 \mathrm{~g}$. More interestingly, the maximum throughput increases roughly linearly with $M$. Such scalability provides strong incentives for further investigations on engineering and implementation details of the system in the future.

This paper also paves the way for further improvement of the baseline MPR system by outlining two possible enhancement schemes. Our initial results indicate that although the baseline MPR system is scalable, it is far from optimal, and higher throughputs can be easily "squeezed out" by incorporating simple enhancements. A fundamental study on the ultimate system throughput limit that can be achieved by different variations of MPR MAC protocols is a fertile ground for future research.

\section{REFERENCES}

[1] B. P. Crow, I. Widjaja, L. G. Kim, and P. T. Sakai, "IEEE 802.11 wireless local area networks," IEEE Commun. Mag., vol. 35, no. 9, pp. 116-126, Sept. 1997.

[2] Y. Xiao and J. Rosdahl, "Performance analysis and enhancement for the current and future IEEE 802.11 MAC protocols," ACM SIGMOBILE Mobile Computing and Communications Review, vol. 7, no. 2, pp. 6-19, Apr. 2003

[3] S. Ghez, S. Verdu, and S.C. Schwartz, "Stability properties of slotted Aloha with multipacket reception capability," IEEE Transactions on Automatic Control, vol. 33, no. 7, pp. 640-649, July 1988.

[4] L. Tong, Q. Zhao, and G. Mergen, "Multipacket reception in random access wireless networks: From signal processing to optimal medium access control," IEEE Commun. Mag., vol. 39, no. 11, pp. 108-112, Nov. 2001.

[5] Q. Zhao and L. Tong, "A dynamic queue protocol for multi-access wireless networks with multipacket reception," IEEE Trans. on Wireless Comm. , vol. 3, no. 6, pp. 2221-2231, Nov. 2004.

[6] C.B. Papadias, A.J. Paulraj, "A constant modulus algorithm for multiuser signal separation in presence of delay spread using antenna arrays," IEEE Signal Processing Letters, vol. 4, no. 6, pp. 178-181, June 1997.

[7] S. Talwar, M. Viberg, and A. Paulraj, "Blind separation of synchronous co-channel digital signals using an antenna array, Part I: Algorithms," IEEE Trans. on Signal Processing, vol. 44, no. 5, May 1996.

[8] S. Talwar, M. Viberg, and A. Paulraj, "Blind estimation of multiple cochannel digital signals using an antenna array," IEEE Signal Processing Lett., vol. 2, no. 1, pp. 29-31, Feb. 1994.

[9] G. Bianchi, "Performance analysis of the IEEE 802.11 distributed coordination function," IEEE Journal on Selected Areas in Communications, vol. 18, no. 3, pp. 535-547, Mar. 2000. 